\title{
El estado del bienestar español: valoración y perspectivas de futuro
}

\section{The Spanish Welfare State: Assessment and Prospects}

\author{
Jorge Calero $^{1}$ \\ Universidad de Barcelona (España)
}

ORCID: https://orcid.org/0000-0001-8443-5412

Recibido: 31-05-2021

Aceptado: 12-06-2021

\section{Resumen}

El artículo tiene como objetivo describir el estado del bienestar español y valorar sus problemas y perspectivas. La descripción de sus características, basada en una comparativa con los países europeos, permite identificar pautas similares a las de otros estados del bienestar meridionales, con una importante subsidiariedad respecto a la familia y una orientación hacia la protección de la población de mayor edad.

Por lo que respecta a la identificación de problemas, se destacan en el artículo dos problemas técnicos. En primer lugar, los relacionados con la sensibilidad ante las crisis económicas, que se traduce, específicamente, en un elevado nivel de desempleo estructural. Y, en segundo lugar, los vinculados a los muy acelerados

\footnotetext{
${ }^{1}$ (jorge.calero@ub.edu). Catedrático de Economía Aplicada en la Universidad de Barcelona. Es Licenciado y Doctor en Ciencias Económicas por la Universidad Autónoma de Barcelona y Master por la Universidad de Londres. Está especializado en Economía de la Educación, campo en que es autor de más de un centenar de artículos en revistas especializadas y diversos libros. Ha sido presidente del Consejo Superior de Evaluación de Cataluña. Ha sido investigador principal de diversos Proyectos nacionales de I+D. Actualmente es investigador principal en el Proyecto de $\mathrm{I}+\mathrm{D}$ "Identificación de políticas educativas eficaces en la mejora de la calidad educativa" e investigador principal en la Red de Excelencia "Análisis económico de las políticas educativas" (Ministerio de Economía y Competitividad). Entre las publicaciones cabe citar: Calero, Jorge y J. Oriol Escardibul (2020) "Teacher quality and student skill acquisition. An analysis based on PIRLS-2011 outcomes". Educational Studies; Calero J., Murillo Huertas I.P., Raymond Bara J.L. (2019) "Education, age and skills: An analysis using PIAAC data". European Journal of Education, vol. 54, n. 1, pp. 72-92; Calero, J., Choi, Á. (2017). "The distribution of skills among the European adult population and unemployment: a comparative approach". European Journal of Education, vol. 52, n. 3, pp. 348-364.
} 
cambios demográficos, que provocan un envejecimiento de la población que agrava las dificultades de financiación del sistema.

Finalmente, el artículo presta atención a diversos problemas políticos y de diseño del estado del bienestar en España. La polarización política y el clientelismo dificultan la toma de decisiones consensuadas destinadas a dar continuidad y legitimidad al estado del bienestar. Se señalan, también, los riesgos vinculados a un diseño "de aluvión" caracterizado por la complejidad y falta de coherencia en el catálogo de prestaciones.

Palabras-clave: estado del bienestar, sector público español, crisis del estado del bienestar, gasto público en protección social.

\begin{abstract}
The article aims at describing the Spanish welfare state and assessing its problems and perspectives. The description of its characteristics, based on a comparison with European countries, makes it possible to identify patterns similar to those of other Southern welfare states, with an important subsidiarity with regard to the family and an orientation towards the protection of the older population.

Regarding the identification of problems, two technical problems are highlighted in the article. First, those related to sensitivity to economic crises, which specifically translates into a high level of structural unemployment. And, second, those linked to the very accelerated demographic changes, which cause an aging of the population that aggravates the difficulties of financing the system.

Finally, the article pays attention to several political and design problems of the welfare state in Spain. Political polarization and clientelism make it difficult to make consensual decisions aimed at giving continuity and legitimacy to the welfare state. The risks linked to a 'patchwork design' characterized by complexity and lack of coherence in the catalog of services are also pointed out.
\end{abstract}

Keywords: welfare state, Spanish public sector, welfare state crisis, government expenditure on social protection.

\title{
1. Introducción
}

El objetivo de este artículo consiste en describir y valorar la situación actual y las perspectivas del estado del bienestar en España. El estado del bienestar está formado por todas las intervenciones del sector público destinadas a cumplir dos funciones: por una parte, el aseguramiento y la protección ante 
riesgos y, por otra, la redistribución de los recursos de tal modo que se alcance un mayor nivel de equidad (estando este definido en cada momento a través de procesos sociales y políticos).

El estado del bienestar español se construyó, en su origen, siguiendo un modelo corporativista, basado en la protección de los trabajadores y en las cotizaciones de éstos a la Seguridad Social. Algunos elementos iniciales en este sentido fueron la Ley de Accidentes de Trabajo de 1910 y el Retiro Obrero, de 1919. En el mismo sentido actuaron, durante la II República, la prestación de desempleo introducida con la Caja Nacional contra el paro forzoso y, durante los primeros años del franquismo, el Seguro de Enfermedad (1942) y el Seguro Obligatorio de Vejez e Invalidez (SOVI) (1947) y, posteriormente, en 1966, la Ley General de la Seguridad Social.

Este modelo básico se fue alterando, a partir de la década de 1980, con nuevos elementos que aportaban protección a ciudadanos con independencia de su participación en el mercado de trabajo. Los ejemplos más relevantes son el establecimiento de un Sistema Nacional Salud en 1986 y, a partir de 1990, la incorporación de las prestaciones no contributivas al sistema de Seguridad Social.

Se trata de un estado del bienestar "de aluvión”, con una diversidad de elementos que se han ido incorporando con el paso de los años y con problema de coherencia interna. La subsidiariedad con respecto a la familia que se observa en muchos de sus componentes lo hace fácilmente identificable con el modelo "meridional" descrito por Ferrera (1986). Los altos niveles de desempleo que caracterizan a la economía española incluso en periodos de crecimiento económico, junto con la debilidad de los ingresos impositivos, hacen difícil su sostenibilidad. La crisis fiscal generada por la Gran Recesión expuso con crudeza estas vulnerabilidades.

La revisión de las características del estado del bienestar español y sus problemas que planteo en este artículo se divide en los siguientes apartados. En el apartado 2 presentaré una descripción de las intervenciones en materia de estado de bienestar en España, centrándome esencialmente en el gasto en protección social; en esta descripción utilizaré comparaciones con diferentes países de la Unión Europea. En el apartado 3 me centraré en las diferentes funciones del estado del bienestar (sanidad, vejez, vivienda, etc.) con objeto de definir la orientación del estado del bienestar español en cuanto a sus actividades y usuarios. El apartado 4 se destina a efectuar una revisión de los problemas más importantes del estado del bienestar en España, aquellos que le impiden cumplir con suficiente eficacia su función cohesionadora y protectora. En el apartado 5 se presentan las conclusiones del artículo. 


\section{Situación y evolución del estado del bienestar en España y en Europa}

Un indicador que nos proporciona una primera aproximación a la magnitud relativa del estado del bienestar en España es el que aparece en el gráfico n. 1, el porcentaje que supone el gasto público en protección social sobre el PIB. El valor correspondiente a España, 23,1\%, se sitúa claramente por debajo de la media de la Unión Europea (26,7). El valor español está cercano al de otros países meridionales, como Grecia o Portugal, aunque otro país meridional como Italia presenta un valor más elevado, por encima de la media europea. Los países nórdicos, junto con otros continentales como Francia, Alemania, Austria, Bélgica y Holanda, son los que presentan los valores más elevados, en torno al 30\% del PIB. En el otro extremo del gráfico, con los valores más bajos, se sitúan, además de Irlanda, el conjunto de países de Europa del Este que habían tenido regímenes socialistas.

El gráfico n. 2 nos ofrece una información más precisa de cómo afecta el estado del bienestar a los ciudadanos, en tanto que se corrige la información del gráfico n. 1 mediante tres elementos: el tamaño poblacional, la magnitud del PIB y las diferencias de precios. Para ello se utilizan valores per cápita y valores monetarios corregidos mediante paridades de poder de compra; los valores resultantes indican los recursos que llegan efectivamente (y con posibilidad de ser comparados) a los ciudadanos. Puede verse cómo tres países con valores de PIB per cápita muy elevados (Luxemburgo, Suiza y Noruega) son los que asignan a cada persona más recursos vinculados al estado del bienestar. El valor de España, 6.456 euros corregidos, sigue siendo considerablemente más reducido a la media europea (8.709).

Gráfico n. 1. Gasto en protección social como porcentaje del PIB. Países de la Unión Europea, EFTA y Reino Unido. 2018.

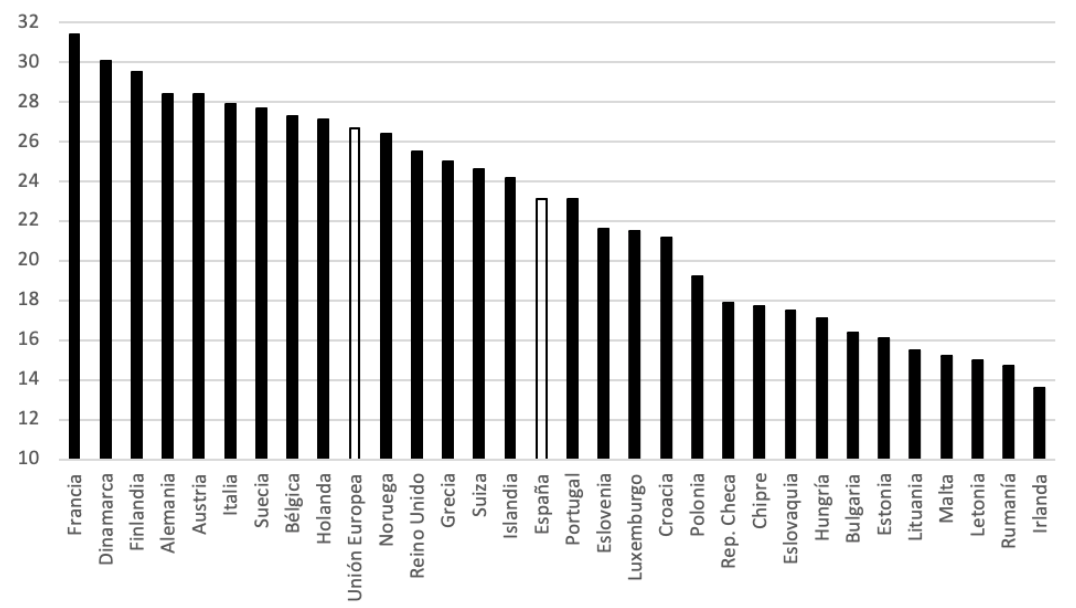

Araucaria. Revista Iberoamericana de Filosofia, Politica, Humanidades y Relaciones Internacionales, año $23, \mathrm{n}^{\circ} 47$. Segundo cuatrimestre de 2021. Pp. 457-478. ISSN 1575-6823 e-ISSN 2340-2199 https://dx.doi.org/10.12795/araucaria.2021.i47.20 
Fuente: elaboración propia a partir de datos de EUROSTAT.

Gráfico n. 2. Gasto en protección social en euros per cápita (paridades de poder de compra). Países de la Unión Europea, EFTA y Reino Unido. 2018.

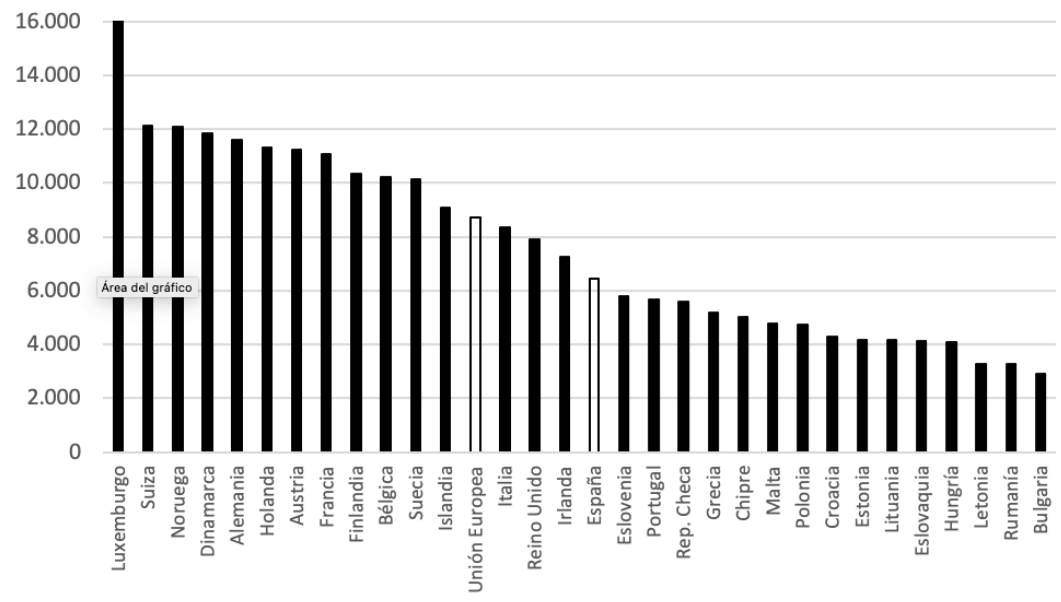

Fuente: elaboración propia a partir de datos de EUROSTAT.

Se han seleccionado, en el gráfico n. 3, cinco países, más la media de la Unión Europea, para describir la evolución del gasto en el estado del bienestar desde antes de la Gran Recesión hasta el periodo más reciente para el que disponemos de datos. En el gráfico se puede observar que la llamada política de austeridad, impuesta en buena medida por las instituciones internacionales que apoyaron financieramente la salida de la crisis, interrumpe en España abruptamente el crecimiento del gasto que se había producido hasta 2010 . Este crecimiento previo había sido intenso durante los años anteriores a 2007 y se intensifica especialmente en 2008 y 2009 , como resultado de las políticas fiscales expansivas que plantea el Gobierno español (al comienzo del segundo mandato del presidente Rodríguez-Zapatero). En 2008, cuando ya resulta innegable la caída de la actividad, el incremento del desempleo generalizado, el fin de la burbuja inmobiliaria y la consiguiente crisis financiera, se incrementa el gasto público en general y, en particular, el vinculado a la protección social. El déficit público, financiado mediante la emisión de deuda, se incrementa considerablemente en ese período: a título de ejemplo, el déficit público se eleva hasta el 11,3\% del PIB en 2009 y la deuda pública en circulación pasa de un $39,7 \%$ del PIB en 2008 a un $60,5 \%$ del PIB en 2010. Las series completas de déficit y deuda pública en circulación aparece en el gráfico n. 4 . 
Este proceso es interrumpido abruptamente en mayo de 2010, momento en el que el Gobierno, presionado por la Unión Europea, abandona la política fiscal expansionista, basada en los estímulos, y empieza a aplicar una serie de medidas de recorte del gasto público, entre las que destaca la reducción de los salarios de los trabajadores públicos. En el gráfico se aprecia cómo entre 2010 y 2014 los valores de gasto en protección social se estabilizan, de una forma similar a lo que sucede en Italia. En otros países de la Unión Europea (Francia, Suecia, Alemania, Reino Unido) el periodo de contención del gasto es más breve, y concluye en 2011.

Una especificidad que afecta severamente a las posibilidades del gasto público en España es la crisis de la deuda pública, que se agrava en 2011, momento en el que la prima de riesgo de la deuda pública española asciende hasta 380 puntos, dificultando extraordinariamente la financiación del déficit público. En agosto de 2011 es aprobada la reforma del artículo 135 de la Constitución, que somete el déficit público a los límites establecidos por la Unión Europea. La situación se convierte en insostenible en julio de 2012 , cuando la prima de riesgo alcanza los 540 puntos y la financiación y el déficit pasa a someterse a un control exhaustivo por parte de la llamada "Troika" (Comisión Europea, Banco Central Europeo y Fondo Monetario Internacional). Los Programas de Estabilidad para los años siguientes a 2012 establecieron objetivos de déficit público descendentes, desde un 6,5\% en 2013 hasta un $3,6 \%$ en 2016 y un $1,6 \%$ en 2018 .

En todo caso, no puede afirmarse que las políticas de "austeridad" fueran generalizadas ni, tampoco, que se prolongaran durante un largo periodo del tiempo. Al contrario, los recortes de gasto más intensos se produjeron sólo en algunos países europeos, particularmente España, Italia, Grecia, Portugal e Irlanda e, incluso en esos países, la recuperación del crecimiento del gasto público había comenzado ya en 2014. Para el conjunto de la Unión Europea, la línea del gráfico n. 3 marca una tendencia con pendiente prácticamente constante, que lleva el gasto desde 6.156 euros corregidos en 2005 hasta 8.586 euros corregidos en 2018. Esta tendencia no permite hablar de la "austeridad" como fenómeno más relevante de la evolución del estado del bienestar en la Europa de la década de 2010. 
Gráfico n. 3. Evolución del gasto en protección social en euros per cápita (paridades de poder de compra). Diversos países. 2005-2018.

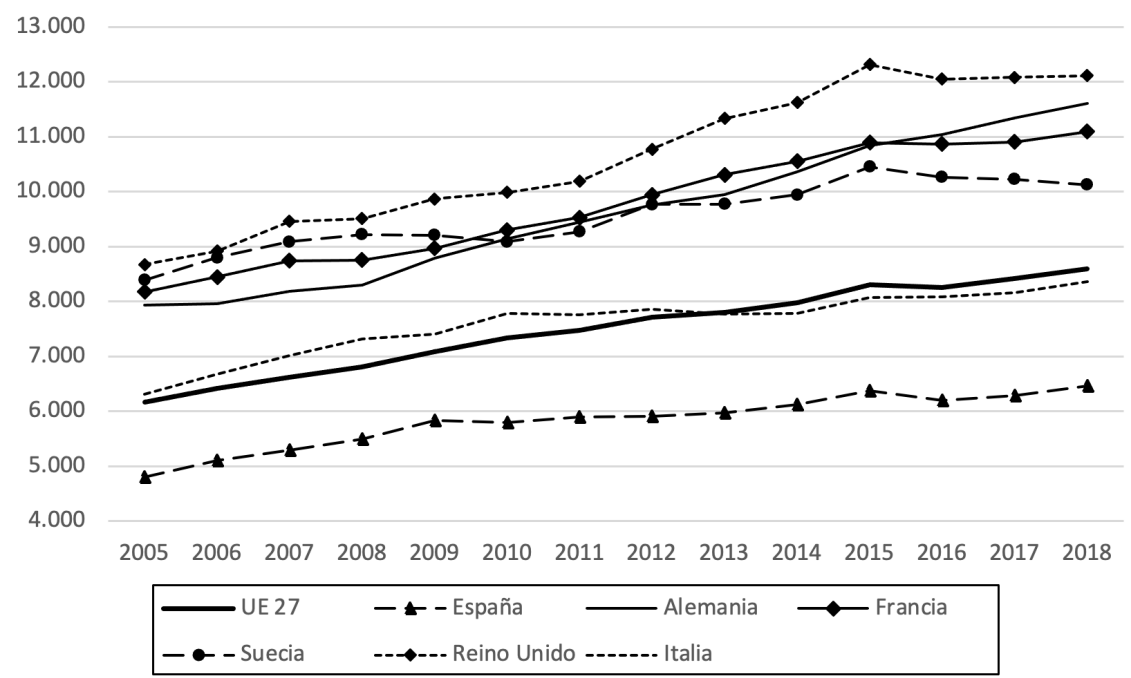

Fuente: elaboración propia a partir de datos de EUROSTAT.

Gráfico n. 4. Déficit y deuda pública en circulación, como porcentajes del PIB. España, 1995-2020.

\section{Déficit (\% del PIB)}

\section{Deuda pública (\% del PIB)}

14

12

12

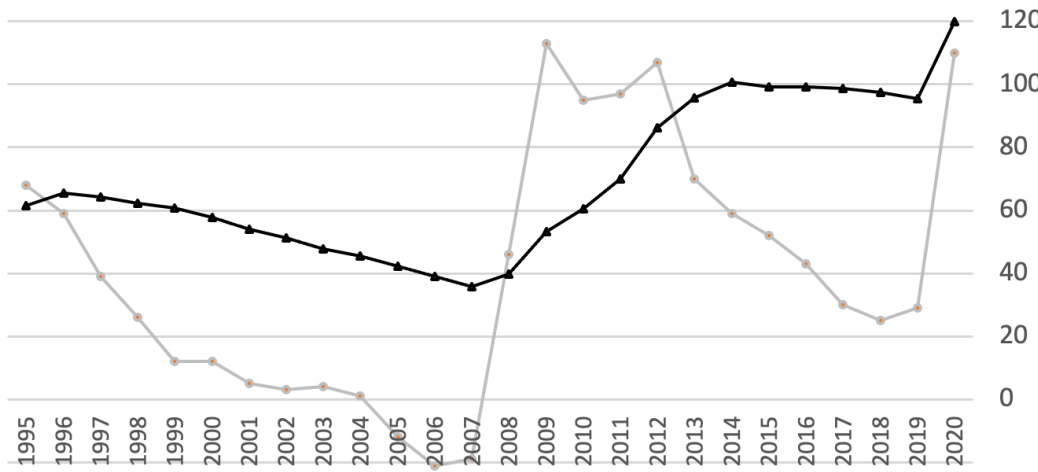

$-4$

$$
\longrightarrow \text { déficit } \longrightarrow \text { deuda pública }
$$

Fuente: elaboración propia a partir de datos de EUROSTAT. 
La evolución histórica de los estados del bienestar ha ido configurando formas de intervención diferenciadas. Uno de los ejes principales de esta diferenciación está constituido por el tipo de intervención, que puede ser en forma de transferencias económicas o en forma de prestación de servicios. De forma general, los estados del bienestar más próximos al modelo universalista proporcionan una mayor proporción de prestaciones en forma de servicios, como los sanitarios, la educación infantil de primer ciclo, los servicios domiciliarios o residenciales para los ancianos, etc. Por el contrario, los estados del bienestar continentales y meridionales en Europa tienden a destinar una mayor proporción de recursos a prestaciones económicas. Estas pautas se pueden observar en el gráfico n. 5, donde vemos que España dedica más de dos tercios de sus recursos del estado del bienestar (69,3\%) al pago de prestaciones económicas, una proporción mayor a la media de la Unión Europea $(65,4 \%)$. Esta situación viene explicada por la importancia relativa de prestaciones dirigidas a la población de mayor edad (pensiones de jubilación) y a las personas desocupadas y al desarrollo tardío, en España, de prestaciones en forma de servicio. Considérese, por ejemplo, cómo incluso en la Ley de Dependencia de 2006 se canalizó buena parte de las ayudas a prestaciones monetarias. La situación es muy diferente en los países nórdicos: todos ellos presentan proporciones de gasto en servicio superiores al $40 \%$. Resulta también notable cómo en Francia y Alemania se ha incrementado en los últimos años la proporción de prestaciones efectuadas en forma de servicio.

La última característica que revisaré en este apartado corresponde a las fuentes de financiación del estado del bienestar. Las dos fuentes esenciales son, por una parte, las cotizaciones a la Seguridad Social (tanto la cuota patronal como la cuota obrera y las cotizaciones de los trabajadores autónomos) y, por otra, la financiación procedente de impuestos generales. La participación de ambas fuentes de financiación proporciona una información sumamente interesante acerca del carácter de un estado del bienestar. Una elevada participación de las cotizaciones a la Seguridad Social se corresponde con sistemas cuyo objetivo central es la protección de los trabajadores y donde ésta se logra a través de la adquisición de derechos contributivos. Se trata de los sistemas encuadrables en los modelos continental y meridional, derivados ambos del "modelo Bismarck" (véase Esping-Andersen, 1990). Por el contrario, la menor participación de las cotizaciones y su sustitución por impuestos generales identifica a sistemas que pretenden proteger al conjunto de la ciudadanía con independencia de su participación en el mercado de trabajo. En el caso español, por ejemplo, cuando en el año 1990 se establecieron los componentes no contributivos en las prestaciones de la Seguridad Social (pensiones no contributivas de jubilación e invalidez) se fijó una participación del Estado en el presupuesto de la Seguridad Social que supuso, inicialmente, en torno a un $30 \%$ del presupuesto. 
El gráfico n. 6 nos permite comprobar que España se sitúa en una posición muy similar a la media europea, con unos gastos en protección social que se financian aproximadamente al $60 \%$ con cotizaciones sociales y al $40 \%$ con impuestos (aportaciones de los presupuestos del Estado, de las Comunidades Autónomas y de las corporaciones locales). Aparte de la evidente posición de los países nórdicos, con una baja participación de las cotizaciones sociales, merece la pena destacar la posición de Francia y Alemania, países que, pese a sus avances hacia estados del bienestar muy extensos y con una presencia elevada de los servicios, siguen manteniendo una alta participación de las cotizaciones sociales en su financiación

Gráfico n. 5. Distribución del gasto en protección social según su asignación en efectivo o en servicio. Países de la Unión Europea, EFTA y Reino Unido. 2018.

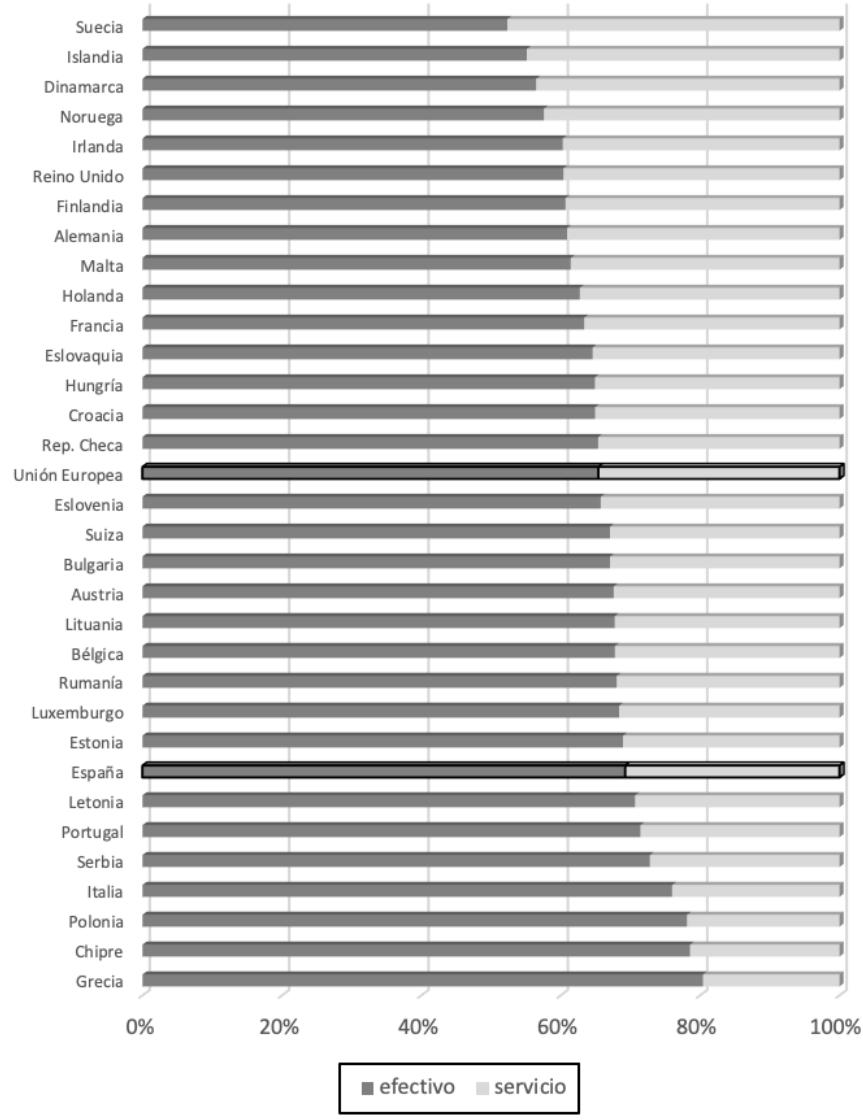


Fuente: elaboración propia a partir de datos de EUROSTAT.

Gráfico n. 6. Financiación del gasto en protección social (impuestos y cotizaciones a la Seguridad Social) como porcentaje del total del gasto. Países de la Unión Europea, EFTA y Reino Unido. 2018.

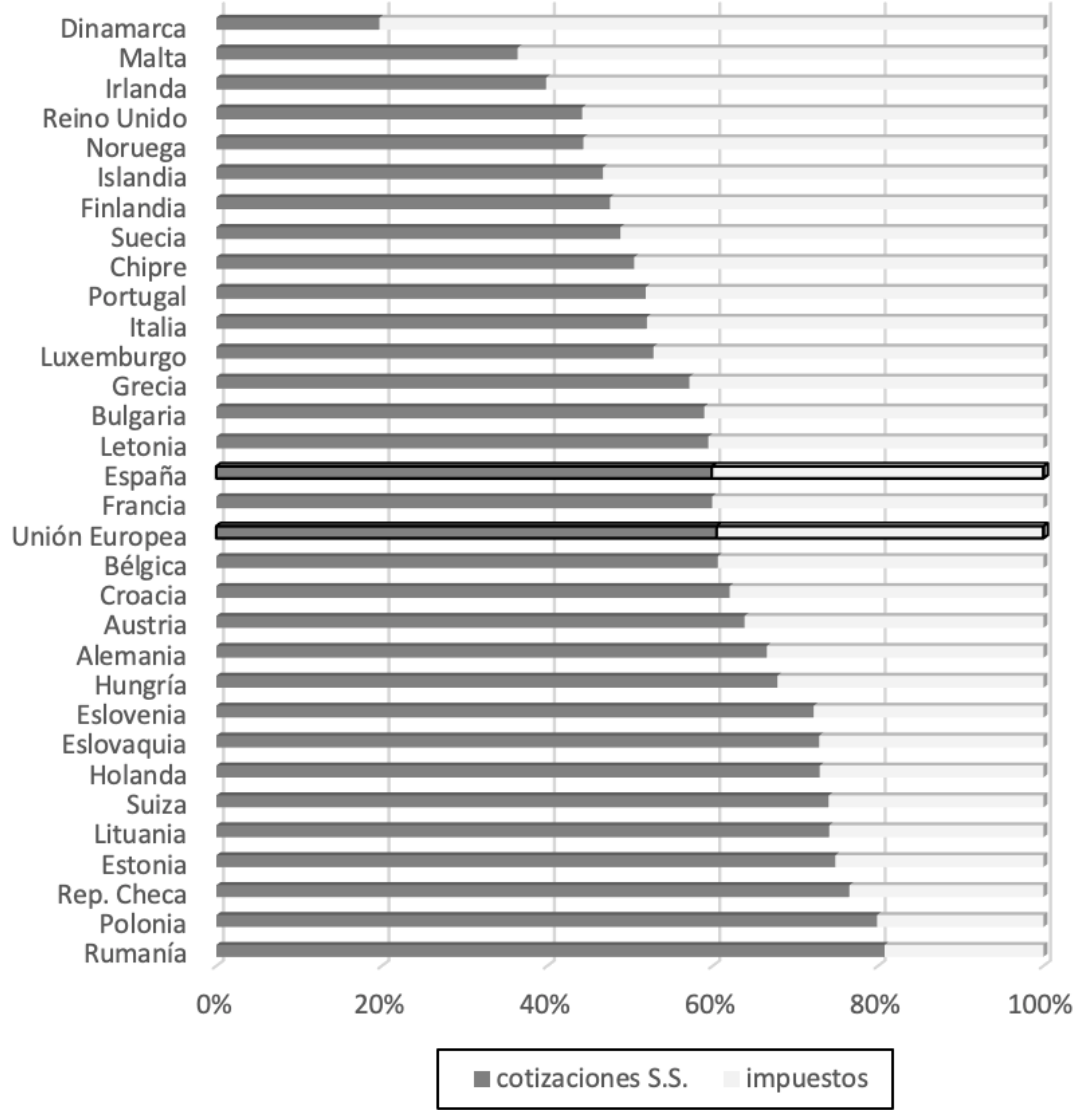

Fuente: elaboración propia a partir de datos de EUROSTAT.

\section{Funciones de protección y redistribución en el estado del bienestar español}

En el cuadro n. 1 aparece el esfuerzo presupuestario que se efectúa, en los países europeos, en las ocho funciones de protección social que utiliza EUROSTAT, la agencia estadística de la Unión Europea. Hay que tener en cuenta que estos datos nos proporcionan una aproximación limitada a la magnitud de 
la intervención pública, en tanto que un mismo esfuerzo presupuestario (en proporción del PIB) puede traducirse en intervenciones muy diferentes no sólo en función de la magnitud del PIB sino, también, en función de la eficiencia con la que se gasten los recursos y en función del efecto de otros inputs que afectan a los resultados del servicio. Sirva de ejemplo, en este sentido, cómo en España, con un nivel de gasto sanitario contenido (6,2\% del PIB) se consiguen resultados en el terreno de la salud, como la esperanza de vida, por ejemplo, comparativamente mejores a los de otros países con esfuerzos presupuestarios superiores. En el ejemplo propuesto un input complementario importante es, entre otros, la dieta mediterránea, que tiene efectos positivos sobre el estado de salud de las personas.

Si bien en el apartado 2 habían quedado patentes una serie de diferencias entre los estados del bienestar europeos, que permitían identificar modelos diferenciados, con los datos del cuadro n. 1 se ponen de relieve estructuras todavía más claras. Una de ellas, a la que quisiera dar énfasis, es la relacionada con la edad de los beneficiarios de las prestaciones en los diferentes países. En el caso español, como en el de otros países meridionales como Italia, Grecia o Portugal, las funciones a las que se destinan proporcionalmente más recursos son las dirigidas a personas de más edad. Podemos ver cómo a la función "vejez", que incluye prestaciones mayoritariamente en efectivo (pensiones de jubilación) pero también prestaciones en servicio, se le dedica en España el 9,8\% del PIB, una cuantía que está ligeramente por debajo de la media europea pero que, sin embargo, es relativamente alta teniendo en cuenta el bajo nivel de gasto en protección social español. Lo mismo sucede, como hemos dicho, para otros países meridionales $\mathrm{y}$, también, para países como Francia o Austria.

Una segunda función, la de "Enfermedad/salud", que recoge mayoritariamente gasto en servicios de sanidad, pero también prestaciones económicas de la Seguridad Social por incapacidad laboral, presenta en España un valor $(6,2 \%$ del PIB) por debajo de la media europea, pero elevado en proporción al nivel de gasto español. Se trata de una función cuyas prestaciones benefician en una proporción muy elevada a la población de mayor edad, como se puede comprobar en Blanco et al. (2012). La asistencia sanitaria se estableció como un servicio de acceso universal a partir de la Ley General de Sanidad de 1986, sustituyendo a un sistema cuyo acceso estaba regulado por la participación en el mercado de trabajo. Se trata de un sistema cuyos costes crecieron rápidamente durante los primeros años del siglo XXI, hasta 2007, para luego contenerse como resultado de las políticas restrictivas aplicadas a partir de 2010. La pandemia del coronavirus, en 2020 y 2021, ha generado un elevadísimo nivel de exigencia al sistema sanitario y ha justificado una expansión de los recursos que a él se destinan. 
Si establecemos comparaciones entre países, podemos ver cómo los países nórdicos, con estados del bienestar muy extensos, presentan todos ellos valores de gasto sanitario sobre el PIB por debajo de la media europea. No parecen estar "especializados" en una función que, característicamente, está orientada a la población de más edad. Son tres países continentales, como Alemania, Holanda y Francia, junto con el Reino Unido, los que destacan con valores muy por encima de la media europea.

Es en la función de "familia/niños" en la que se aprecia mejor la diferencia a la que estoy apuntando: con una media europea de gasto del 2,2\% del PIB, en los países del Sur de Europa nos encontramos con valores muy bajos, como el 1,3\% de España, o el 1,1 o 1,2 de Italia y Portugal, respectivamente. Sin embargo, todos los países escandinavos presentan valores en torno o superiores al 3\% del PIB. A esta función se han incorporado en los últimos años, con niveles de gasto también muy elevados, Alemania y Francia.

La protección vinculada al desempleo incorpora, en el cuadro n. 1, tanto las prestaciones económicas a los desempleados como las políticas activas del mercado de trabajo (formación y orientación, principalmente). España dedicó en 2018 un 1,7\% de su PIB a este tipo de protección, un nivel sólo por debajo del gasto efectuado por Bélgica, Francia y Finlandia, entre los países comparados. El gasto en protección al desempleo depende, como es lógico, tanto de la incidencia del desempleo como de la intensidad del nivel de protección. La incidencia del desempleo en España es la segunda más alta de la Unión Europea: un 15,3\% de la población activa en 2018, sólo por detrás de Grecia (con un 19,3\%), cuando la media de la Unión Europea era del 7,1\%; téngase en cuenta, además, que 2018 fue un año de crecimiento económico, que daba continuidad a la fase de recuperación posterior a la Gran Recesión. El porcentaje tan elevado del gasto en protección por desempleo en España viene explicado, por tanto, por la incidencia del desempleo más que por la intensidad de la protección. Por lo que respecta a los tipos de protección, el 91,2\% de las prestaciones se canaliza en España a transferencias monetarias a los desempleados. La importancia de las prestaciones económicas con relación a las políticas activas se mantiene en la mayor parte de los países de la Unión. En la media, este porcentaje supone un 93,5\% y en países diversos pero que comparten un nivel muy elevado de gasto también se mantiene esta pauta: es el caso de Francia, con un 95\% y Finlandia, con un 91\%.

El gasto social en vivienda es otra de las zonas donde se plasman claramente las diferencias en los modelos de protección. España presenta un nivel de gasto muy bajo, de únicamente el $0,1 \%$ del PIB, cuando la media europea es del $0,4 \%$. El gasto en vivienda en España se canaliza mayoritariamente, además, a través de gasto fiscal (tipos reducidos del IVA y deducciones por compra y alquiler de vivienda). Otros países meridionales, como Portugal, Grecia e Italia, 
presentan niveles de gasto en esta función también muy reducidos o nulos. En otros países, las prestaciones vinculadas a la vivienda son mucho más intensas. Es el caso del Reino Unido, los países escandinavos (con la excepción de Noruega) y, también Francia.

Finalmente, en la función de "Exclusión social" se recoge una serie de prestaciones, en su mayor parte correspondientes a programas de mantenimiento de rentas como las Rentas Mínimas de Inserción, las Rentas Activas de Inserción y, más recientemente, el Ingreso Mínimo Vital. En el caso español estos programas se iniciaron a finales de la década de 1980 en algunas comunidades autónomas, con las Rentas Mínimas de Inserción, financiadas y gestionadas por las comunidades autónomas y, por tanto, con diversidad de condiciones de acceso, cuantías y duración ${ }^{2}$. Los programas de mantenimiento de rentas en España están caracterizados por la fuerte comprobación de los ingresos de los hogares. Puede verse en el cuadro n. 1 cómo el gasto en esta función es en España comparativamente reducido, únicamente un 0,2\% del PIB cuando la media europea es del 0,6\%. De nuevo los países escandinavos, pero también Francia y Holanda, dedican grandes cantidades de recursos a esta función.

Cuadro n. 1. Gasto en protección social, según funciones, como porcentaje del PIB. Países de la Unión Europea, EFTA y Reino Unido. 2018.

\begin{tabular}{|c|c|c|c|c|c|c|}
\hline $\begin{array}{l}\text { Enfermedad/ } \\
\text { Salud }\end{array}$ & Discapa-cidad & Vejez & $\begin{array}{c}\text { Supervi- } \\
\text { vientes }\end{array}$ & $\begin{array}{l}\text { Familia/ } \\
\text { niños }\end{array}$ & Desempleo & Vivienda \\
\hline
\end{tabular}

$\begin{array}{lrrrrrrrr}\text { Unión } & & & & & & & & \\ \text { Europea } & \mathbf{7 , 8} & \mathbf{2 , 0} & \mathbf{1 0 , 7} & \mathbf{1 , 6} & \mathbf{2 , 2} & \mathbf{1 , 2} & \mathbf{0 , 4} & \mathbf{0 , 6} \\ \text { Bélgica } & 7,4 & 2,4 & 11,0 & 1,7 & 2,0 & 1,8 & 0,2 & 0,7 \\ \text { Bulgaria } & 4,9 & 1,2 & 7,1 & 0,9 & 1,7 & 0,5 & 0 & 0,2 \\ \text { Rep. Checa } & 6,0 & 1,1 & 7,8 & 0,6 & 1,7 & 0,4 & 0,2 & 0,2 \\ \text { Dinamarca } & 6,5 & 4,5 & 12,0 & 0,2 & 3,4 & 1,3 & 0,7 & 1,5 \\ \text { Alemania } & 10,0 & 2,5 & 9,2 & 1,7 & 3,3 & 0,9 & 0,5 & 0,3 \\ \text { Estonia } & 4,8 & 1,8 & 6,5 & 0,0 & 2,3 & 0,5 & 0,1 & 0,1 \\ \text { Irlanda } & 5,3 & 0,8 & 4,3 & 0,4 & 1,2 & 1,0 & 0,5 & 0,1 \\ \text { Grecia } & 4,9 & 1,0 & 13,6 & 2,4 & 1,7 & 0,9 & 0 & 0,5 \\ \text { España } & \mathbf{6 , 2} & \mathbf{1 , 6} & \mathbf{9 , 8} & \mathbf{2 , 3} & \mathbf{1 , 3} & \mathbf{1 , 7} & \mathbf{0 , 1} & \mathbf{0 , 2}\end{array}$

${ }^{2}$ Una revisión de la situación actual de estas ayudas, detallada por comunidades autónomas, puede encontrarse en Ministerio de Derechos Sociales y Agenda 2030 (2020). 


\begin{tabular}{|c|c|c|c|c|c|c|c|c|}
\hline Francia & 9,0 & 2,0 & 12,7 & 1,7 & 2,4 & 1,9 & 0,7 & 1,0 \\
\hline Croacia & 7,1 & 2,2 & 7,2 & 1,8 & 1,9 & 0,6 & 0 & 0,3 \\
\hline Italia & 6,4 & 1,6 & 13,7 & 2,6 & 1,1 & 1,5 & 0 & 0,9 \\
\hline Chipre & 3,4 & 0,8 & 8,5 & 1,3 & 1,2 & 1,0 & 0,3 & 1,2 \\
\hline Letonia & 4,1 & 1,3 & 7,0 & 0,2 & 1,6 & 0,7 & 0,1 & 0,1 \\
\hline Lituania & 4,7 & 1,4 & 6,4 & 0,4 & 1,6 & 0,7 & 0,1 & 0,3 \\
\hline $\begin{array}{l}\text { Luxem- } \\
\text { burgo }\end{array}$ & 5,4 & 2,2 & 6,9 & 1,6 & 3,3 & 1,2 & 0,4 & 0,5 \\
\hline Hungría & 4,8 & 1,0 & 7,6 & 0,9 & 2,0 & 0,3 & 0,5 & 0,1 \\
\hline Malta & 5,3 & 0,6 & 6,6 & 1,2 & 0,8 & 0,3 & 0,1 & 0,2 \\
\hline Holanda & 9,3 & 2,5 & 10,3 & 1,0 & 1,1 & 1,0 & 0,5 & 1,4 \\
\hline Austria & 7,5 & 1,8 & 12,7 & 1,6 & 2,7 & 1,6 & 0,1 & 0,5 \\
\hline Polonia & 4,2 & 1,3 & 9,1 & 1,7 & 2,5 & 0,2 & 0 & 0,1 \\
\hline Portugal & 6,0 & 1,6 & 11,6 & 1,8 & 1,2 & 0,7 & 0 & 0,2 \\
\hline Rumanía & 4,3 & 0,9 & 7,2 & 0,6 & 1,5 & 0,1 & 0 & 0,1 \\
\hline Eslovenia & 7,3 & 1,0 & 9,0 & 1,3 & 1,8 & 0,5 & 0 & 0,7 \\
\hline Eslovaquia & 5,7 & 1,5 & 7,1 & 0,9 & 1,5 & 0,5 & 0,1 & 0,2 \\
\hline Finlandia & 6,7 & 2,8 & 12,7 & 0,8 & 2,9 & 1,8 & 0,9 & 0,8 \\
\hline Suecia & 7,5 & 2,8 & 12,2 & 0,3 & 2,9 & 0,9 & 0,4 & 0,8 \\
\hline Islandia & 8,8 & 3,8 & 7,1 & 0,5 & 2,5 & 0,5 & 0,4 & 0,6 \\
\hline Noruega & 7,5 & 4,3 & 9,9 & 0,2 & 3,1 & 0,5 & 0,1 & 0,7 \\
\hline Suiza & 7,8 & 2,0 & 10,6 & 1,2 & 1,5 & 0,8 & 0,3 & 0,6 \\
\hline $\begin{array}{l}\text { Reino } \\
\text { Unido }\end{array}$ & 8,3 & 1,6 & 11,2 & 0,1 & 2,3 & 0,3 & 1,1 & 0,5 \\
\hline
\end{tabular}

Fuente: elaboración propia a partir de datos de EUROSTAT.

\section{Algunos problemas del estado del bienestar español}

La revisión de la situación del estado del bienestar español presentada en los apartados 2 y 3 ha sido esencialmente descriptiva. En este apartado quisiera discutir algunos de los problemas que está afrontando (y previsiblemente va a afrontar en el próximo futuro) el estado del bienestar español. Algunos de ellos 
son compartidos con el resto de estados del bienestar europeos, especialmente los de los países meridionales; otros afectan con singular gravedad en el caso español. Dividiré estos problemas en dos grupos: en primer lugar, los técnicos y financieros; en segundo lugar, los político-ideológicos y de diseño.

Por lo que respecta a los problemas técnicos y financieros, destacaré especialmente dos: la especial sensibilidad ante las crisis económicas y los cambios demográficos que generan un fuerte envejecimiento de la población. Las crisis económicas se traducen en España en fuertes niveles de desempleo. Incluso en periodos de crecimiento el nivel de desempleo en España es muy elevado, de hecho el más elevado de Europa sólo por detrás de Grecia, como he mencionado anteriormente. Puede verse en el gráfico 7 la diferencia de nivel entre las curvas de España y la media de la Unión Europea, así como la mayor sensibilidad del desempleo en España ante las fases bajas del ciclo económico (obsérvese, especialmente, el periodo comprendido entre 2007 y 2014). Cada crisis profundiza, además, en la separación entre dos zonas del mercado de trabajo español, una de insiders y otra de outsiders (Lindbeck y Snower, 1988), separación que es también de las más acusadas en Europa. Estos problemas del mercado de trabajo repercuten en las necesidades de protección de los hogares.

La mayor protección proporcionada por las familias en momentos de crisis económicas produce, sin embargo, el fenómeno recogido en el gráfico 8: el porcentaje de hogares donde ningún miembro trabaja es elevado en España, pero proporcionalmente las diferencias con respecto a la Unión Europea son mucho más pequeñas en este indicador. La situación se puede resumir indicando que en España es mucho más probable que en el resto de países de la Unión Europea estar desempleado, pero también es mucho más probable que, una vez que se da esa situación, convivas con una familia donde al menos una persona trabaja. Una elevada subsidiariedad con respecto a la familia en el modelo de estado del bienestar español es a la vez causa y consecuencia de este fenómeno.

Por otra parte, en los periodos de crisis económica se combina en España, con especial intensidad, una recaudación fiscal débil con necesidades de gasto crecientes. Téngase en cuenta que la presión fiscal en España, situada en el $35,4 \%$ del PIB en 2019, es menor a la existente como media en los países de la Unión Europea (41,1\%). Los crecimientos de la demanda de prestaciones sociales durante los periodos de crisis con caídas fuertes de la recaudación tienen consecuencias en términos de déficit público y deuda pública que ya quedaron recogidos en el gráfico 4. Las dificultades del estado del bienestar español para cubrir, durante los periodos de crisis económica, las necesidades de protección de los hogares quedan reflejadas en el gráfico 9. En él aparecen, para diversos países de la Unión Europea, las evoluciones del indicador AROPE ("At risk of poverty or social exclusión"). Puede verse cómo los valores del indicador, que ya son en estos dos países más elevados que la media europea, 
crecen especialmente durante el periodo 2009-2014. En países como Francia y Alemania la crisis económica no modificó apenas el riesgo de pobreza o exclusión social de la población.

Gráfico n. 7. Tasa de desempleo. 1993-2020

30

25

20

15

10

5

0

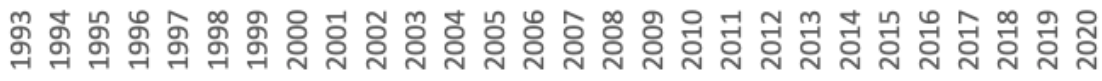

\section{—Unión Europea ——España}

Fuente: elaboración propia a partir de datos de EUROSTAT.

Gráfico n. 8. Porcentaje de hogares donde ningún miembro trabaja.

16

14

12

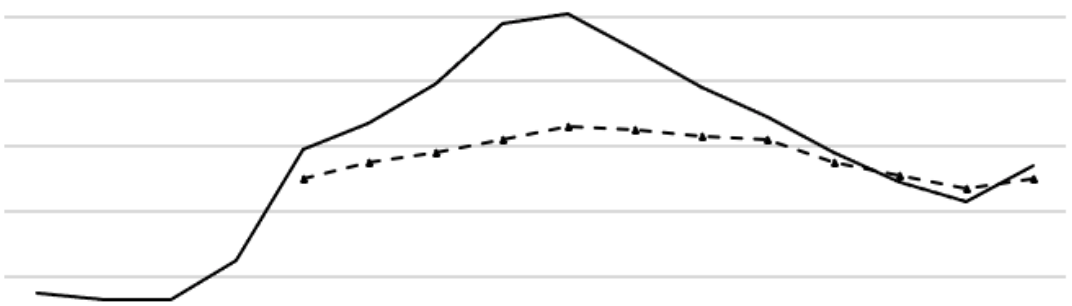

2

0

2005200620072008200920102011201220132014201520162017201820192020

--s- - Unión Europea España

Araucaria. Revista Iberoamericana de Filosofia, Política, Humanidades y Relaciones Internacionales, año $23, \mathrm{n}^{\circ} 47$. Segundo cuatrimestre de 2021. Pp. 457-478. ISSN 1575-6823 e-ISSN 2340-2199 https://dx.doi.org/10.12795/araucaria.2021.i47.20 
Fuente: elaboración propia a partir de datos de EUROSTAT.

Gráfico n. 9. Porcentaje de población en riesgo de pobreza o exclusión social (AROPE). Países de la Unión Europea.

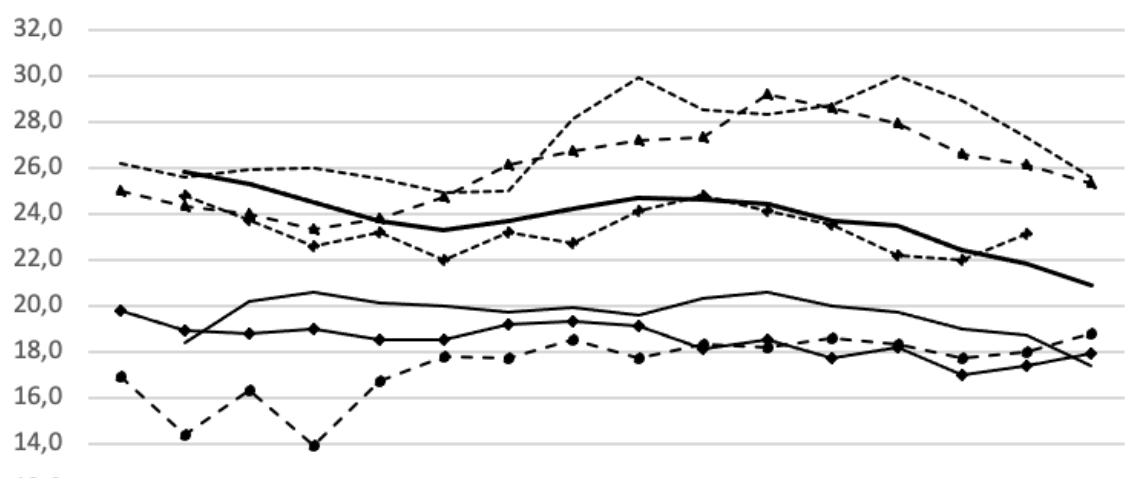

12,0

2004200520062007200820092010201120122013201420152016201720182019

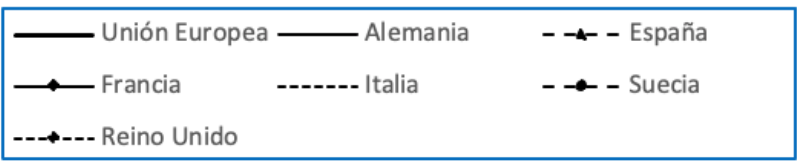

Fuente: elaboración propia a partir de datos de EUROSTAT.

Todavía dentro de la zona de los problemas de carácter técnico, el otro ámbito que impacta decisivamente sobre el estado del bienestar español es el relativo a la demografía. El envejecimiento de la población ha afectado especialmente a Europa y, muy especialmente, a España. El envejecimiento somete a tensiones muy fuertes al estado del bienestar, por la vía del incremento de gasto en pensiones de jubilación y sanidad y, también, por la vía de la contracción de la población potencialmente contribuyente a la Seguridad Social. La evolución de la tasa de fecundidad (número de hijos por mujer) que puede observarse en el gráfico 10 es concluyente: en tan solo veinte años, entre 1974 y 1994, la tasa disminuyó de 2,9 a 1,2 en España, un descenso mucho más rápido que el que se produjo como media en los países de la Unión Europea y, de forma más general, de la OCDE, que habían empezado pocos años antes el descenso. Esta transición tan abrupta estuvo causada en buena medida por la incorporación masiva de la mujer al mercado de trabajo y por la incidencia de la crisis económica sobre las rentas de los hogares. La tasa de fecundidad sólo se recuperaría ligeramente, alcanzando el nivel de 2,4, en el periodo comprendido entre 2000 y 2007, como resultado de la llegada de población inmigrante. Sin embargo, la Gran Recesión provocó nuevas reducciones a partir 
de 2008 que dejaron la tasa en 2,24 en 2019; la pandemia de coronavirus ha provocado nuevas reducciones por el momento no contabilizadas. Unas tasas de fecundidad que no han excedido el nivel de 1,4 en los últimos veinte años condena al envejecimiento rápido de la población y a una dependencia de la inmigración como factor paliativo de la contracción demográfica. Considérese que, en el periodo comprendido entre 1960 y 2018, la esperanza de vida ha pasado en España de 71,6 años para las mujeres y 66,8 años para los hombres a 86,3 y 80,7 , respectivamente, nivel que deja a España entre los países del mundo con mejor resultado en este indicador. Un incremento de 15 años de esperanza de vida en un periodo de menos de cuarenta años es un logro extraordinario que, sin embargo, afecta decisivamente a los ingresos y gastos del estado del bienestar (piénsese en las pensiones de jubilación y en el gasto sanitario) y dificulta enormemente la sostenibilidad del estado del bienestar.

El cambio radical de la forma de la pirámide poblacional provoca un incremento muy rápido de la tasa de dependencia, la relación entre la población mayor de 65 años y la población en edad de trabajar. En el gráfico n. 11 se puede ver cómo la tasa se incrementa constantemente entre 1960 y la actualidad, con un único periodo de estabilidad entre 2000 y 2008 que coincide, nuevamente, con la llegada de población inmigrante. La tasa de dependencia del conjunto de la Unión Europea se sitúa sólo muy ligeramente por encima de la española.

Gráfico n. 10. Tasa de fecundidad (hijos por mujer). 1960-2019.

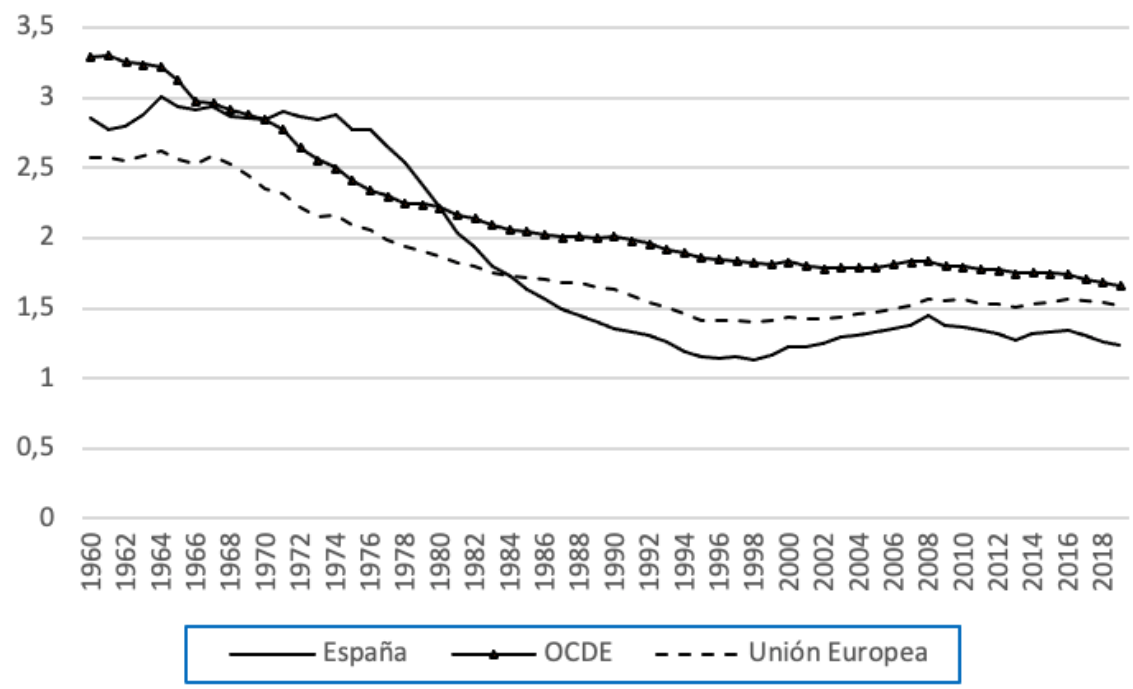

Fuente: elaboración propia a partir de datos del Banco Mundial. 
Gráfico n. 11. Tasa de dependencia. Población de 65 más años como proporción de la población en edad de trabajar (16-65 años). 1960-2019.
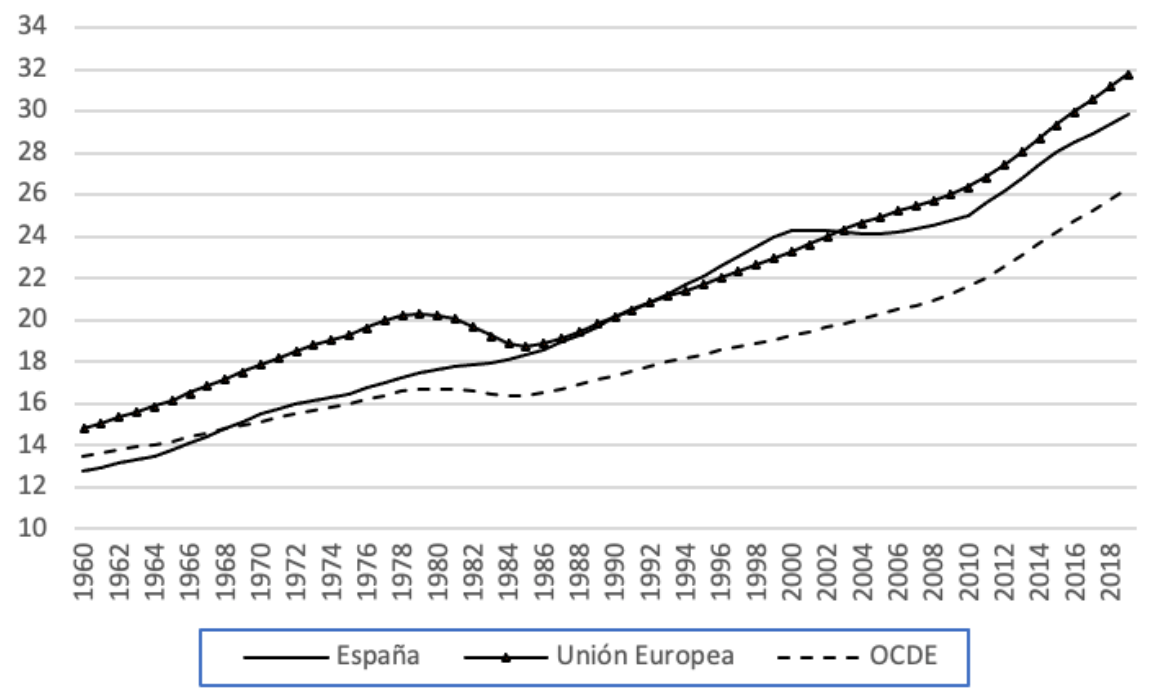

Fuente: elaboración propia a partir de datos del Banco Mundial.

En el segundo grupo de problemas, los político-ideológicos y los relacionados con el diseño, quisiera dar énfasis a los problemas que le generan al estado del bienestar un panorama político muy polarizado. Los retos que debe afrontar el estado del bienestar español son de gran calado, y exigirían un consenso político profundo, un acuerdo de largo plazo sobre reformas y reorientaciones, que es muy poco probable en el escenario político más reciente. El caso que ilustra mejor los efectos de la polarización política lo encontramos en el ámbito de la educación, donde las leyes básicas educativas van sustituyéndose a medida que se sustituyen los gobiernos y donde ha sido imposible en dos ocasiones (2010 y 2018) alcanzar un pacto educativo que tuviera el acuerdo de los principales partidos políticos. También las reformas necesarias para garantizar la sostenibilidad del sistema de pensiones requieren de una cierta contención en la polarización.

Dentro de ese marco de polarización, actualmente, en España, la defensa del estado del bienestar está asociado con la izquierda política, mientras que a la derecha política se le asocia frecuentemente con las políticas de austeridad o de recortes. Para ser justos, conviene señalar que la política de austeridad se inició en 2010, en pleno mandato gubernamental del PSOE; también, que el Partido Popular, al formar gobierno a finales de 2011, no tenía apenas margen para alterar una política fiscal restrictiva, marcada por las exigencias de la Unión 
Europea, el Banco Central Europeo y el Fondo Monetario Internacional. Durante el periodo comprendido entre 2011 y 2018 los gobiernos del Partido Popular estuvieron sometidos a una senda de reducción del déficit público que en modo alguno favorece la expansión del estado del bienestar. El estado de salud del estado del bienestar español dependerá, en los próximos años, de la capacidad de los partidos políticos para evitar que la polarización política bloquee las reformas necesarias para el mantenimiento del estado del bienestar.

La propia polarización incrementa las tendencias al uso clientelista del estado del bienestar. Este uso clientelista se exacerba en el caso de algunos partidos de corte claramente populista. Cuanto más segmentada está la utilización del estado del bienestar más frágil es este ante su utilización clientelista: si las prestaciones se vinculan a grupos poblacionales muy específicos ("los nuestros" para un partido en concreto) estas permiten obtener votos fácilmente de esos grupos poblacionales. Estas prácticas, a la vez que refuerzan a los partidos, debilitan al propio estado del bienestar, en tanto que es visto por parte de la ciudadanía como un instrumento de los rivales políticos. Estos procesos son muy peligrosos para la viabilidad del estado del bienestar, que requiere de una aceptación muy extensa ya que su financiación es tremendamente exigente.

Para finalizar este apartado mencionaré los problemas vinculados al diseño global del sistema del estado del bienestar. Como he dicho anteriormente, el estado del bienestar español es un sistema "de aluvión", formado a lo largo de muchos años por instrumentos no necesariamente coherentes entre sí. Del mismo modo que muchas compañías han optado en los últimos años por las extensiones de línea como estrategia de marketing, por la que, por ejemplo, los sabores y combinaciones de yogures de una misma marca se multiplican continuamente, el estado del bienestar ha sufrido una multiplicación notable de sus prestaciones. Este fenómeno dificulta la comprensión del acceso a estas prestaciones a determinadas capas de la población, incrementando el fenómeno del non take-up (individuos u hogares que no solicitan una prestación a pesar de reunir las condiciones para hacerlo). La complejidad y diversidad de las prestaciones induce, también, al fenómeno que Lindbeck (1988) denominó "estado de transferencias", en donde las transferencias dejan de tener un sentido global (desde los más ricos a los más pobres, por ejemplo) y acaban convirtiéndose en un terreno complejo, donde la búsqueda de rentas y la presión de los lobbies decide el acceso a los recursos. En este proceso, como es lógico, pierde legitimación y apoyo, considerablemente, el estado del bienestar. 


\section{Conclusiones}

Se planteaba como objetivo para el artículo la descripción y valoración de la situación actual y las perspectivas del estado del bienestar en España. Después de una revisión comparativa (con respecto, especialmente, a los países europeos) de sus características, se ha podido identificar el bajo nivel de gasto en protección social, junto con unas pautas de gasto, según sus funciones, que lo acercan al modelo meridional de estado del bienestar, con una importante subsidiariedad con respecto a la familia. Resulta muy destacable la orientación de la protección hacia los grupos poblacionales de más edad, a diferencia de otros estados del bienestar cuyos objetivos están centrados en la promoción y protección de los jóvenes.

Se han valorado, también, en el artículo, los principales problemas y fragilidades del estado del bienestar español. Destaca, en este ámbito, la especial sensibilidad ante las crisis y el elevado nivel de desempleo estructural, que se exacerba en periodos de crisis. La financiación del gasto público tiene en España la dificultad constante del bajo nivel de recaudación, lo que le ha condenado, especialmente desde 2008, a niveles elevados de déficit y de emisión de deuda pública. La crisis provocada por la pandemia de coronavirus, en 2020 y 2021, ha acelerado esta tendencia.

La evolución de las variables demográficas constituye un problema esencial del estado del bienestar español y condiciona su sostenibilidad. La brusca caída de la natalidad desde la década de 1970, junto con el rápido crecimiento de la esperanza de vida, ha provocado cambios radicales en la pirámide poblacional, que continuará estrechándose por la base y ampliándose por la parte superior. Ello ha provocado, y provocará todavía más en el futuro, importantes tensiones derivadas del incremento de los gastos (esencialmente en pensiones de jubilación y en sanidad) y del descenso de los ingresos por cotizaciones sociales e impuestos.

Se ha prestado atención, también, a diferentes problemas políticos y de diseño del estado del bienestar español. En concreto, la polarización política y el incremento de los riesgos de clientelismo y populismo que esta conlleva constituye un importante problema. Las serias amenazas que sufre el estado del bienestar español harían recomendable alcanzar decisiones consensuadas que incorporen a una diversidad de posiciones políticas; este proceso no parece probable en vista de la situación política actual.

Finalmente, he señalado en el artículo los serios problemas de diseño del estado del bienestar español. Su construcción se ha efectuado, como en la mayoría de los países, a partir de piezas superpuestas no siempre compatibles o coherentes entre sí. El riesgo de caer en lo que Lindbeck (1988) denominó "estado de transferencias", en donde la complejidad de las reglas facilita la búsqueda de rentas y la presión de los lobbies, consiste en que el estado del bienestar pierda legitimidad y excluya a amplias zonas de la población. 


\section{Bibliografía:}

Arts, W. y Gelissen, J. (2012) "Three worlds of welfare capitalism or more? A state-of-theart report" Journal of European Social Policy, vol. 12, 2, pp. 137-158

Bandrés (2012) "La aritmética del Estado de bienestar en España” Ekonomiaz, n. 81, pp. $38-61$

Blanco, Ángela; Urbanos, Rosa y Thuissard, Israel J. (2012) Distribución del gasto sanitario público por edad y sexo en España: análisis de la década 1998-2008. Documento de trabajo $n^{\circ}$ 673/2012 Fundación de las Cajas de Ahorros. Madrid: Fundación de las Cajas de Ahorros.

Esping-Andersen, G.(1993) Los tres mundos del Estado del Bienestar. Valencia: Alfons el Mangnànim.

Ferrera M. (1996) The "Southern Model" of Welfare in Social Europe. Journal of European Social Policy, vol. 6, n. 1, pp. 17-37.

Gamble (2018) "El estado de bienestar y las políticas de austeridad”. OpenMind.

Gimeno, Juan Antonio (2008) "Estado de Bienestar: evolución y perspectivas" en El Estado de bienestar ante los nuevos riesgos sociales pp. 31-46.

IMSERSO (2015) 25 años de las pensiones no contributivas de la Seguridad Social. Madrid: IMSERSO.

Lindbeck, Assar (1988) "Consequences of the Advanced Welfare State", The World Economy, vol. 11, n. 1, pp. 19-38.

Lindbeck, Assar y Dennis J. Snower (1988) The Insider-Outsider Theory of Employment and Unemployment, MIT Press, Cambridge, Massachusetts.

Ministerio de derechos sociales y Agenda 2030 (2020) El Sistema Público de Servicios Sociales. Informe de rentas mínimas de inserción año 2019. Madrid: Ministerio de derechos sociales y Agenda 2030.

Moreno, Luis (2013) La Europa asocial. Crisis y estado del bienestar. Barcelona, Ediciones Península

Muñoz de Bustillo (2016) "Crisis, post-crisis y Estado de Bienestar: ¿hacia dónde transita el Modelo Social Europeo?” Cuadernos Europeos de Deusto, núm. 54/2016, págs. 121-154.

Pérez Díaz, J. (2011) "Demografía, envejecimiento y crisis ¿Es sostenible el Estado de Bienestar?" capítulo del libro El Estado de bienestar en la encrucijada: nuevos retos ante la crisis global: Federación de Cajas de Ahorros Vasco-Navarras, pp. 47-62.

Pino, Eloísa del; Rubio, M. J. (dirs.) (2016) Los Estados del Bienestar en la encrucijada. Politicas sociales en perspectiva comparada. Madrid: Tecnos. $2^{\mathrm{a}}$ Edición.

Uroz, Jorge (2010) "La llamada crisis del modelo de estado de bienestar: reestructuración y alternativas”. Miscelánea Comillas, vol. 68, núm. 132. 